\title{
Performance of a Linux-based Network Router
}

\section{Dr. David Border, Bowling Green State University}

David A. Border, Ph.D., holds a principle research interest in electronic information systems. This field includes digital communication and networking and intelligent networked devices. His work includes wireless sensor networks. Prior research included work on signal bandwidth compression and signal specific data encoding techniques. His technology application interest includes networked systems. Typical teaching duties include junior- and senior-level courses in the Electronics and Computer Engineering Technology (ECET) program. Within this course set are the curriculum's networking and communication courses. As is true with his ECET faculty colleagues, Border supports the program with teaching assignments, as needed, in freshman- and sophomore-level courses offerings. Examples of these include the sophomore level electric circuits and digital electronics courses. Border teaches a digital communication graduate course within a Ph.D. Consortium Technology Management program, as well as other graduate level courses at BGSU.

Border served as interim department chair of the Engineering Technologies department. He served as chair of the university Faculty Senate curriculum and academic affairs committee. He is chair of the University Faculty Senate. 


\title{
Performance of a Linux-based Router
}

\begin{abstract}
Today's routers have increasingly short life cycles. The short life cycle is due to the growing demand for bandwidth by users. High traffic under limited bandwidth conditions results in a significant number of dropped connections and excruciatingly slow speeds. Coupled with the practice of sporadic firmware updates for the majority of commercial routers, consumers usually have to purchase new hardware every two years to maintain consistent speeds. This project explores an alternative, building a home-built router suitable for use in senior projects and special projects. While our lab has made use of a "specimen" router built from an aging Dell Computer in the past; while this router has worked quite well when needed; it was decided to initiate a formal work that built home-built routers. The hardware basis of these is the PC. The PC runs a Linux distribution OS. The paper reports the building and configuration steps. The performance of the router is compared to existing devices and reported. Tests of the networks containing the home-built router and commercially built router were done and reported. Objective data-rate tests chosen allowed for the setting of test packet size, test duration, and traffic direction. The work also reports the cost of material for the home-built routers. Since the home-built routers do not implement a pseudo-Cisco IOS, they are not suitable for teaching Cisco Academy content. However, the home-built routers do implement well-understood router functions and capabilities. A review of these implementations appears in the paper for completeness.
\end{abstract}

\section{Introduction}

Based on the first-hand experience of converting an aging mini-tower PC into a network router, it was decided to change to a different form factor PC chassis. A form factor whereby the PC would meet general business criteria, such as being compact, disk-less, and fan-less. The computer used as the server in this project is a product marketed by Qotom [1]. Figure 1 shows the device front and back view. Note the width ( 6 inches) and the depth ( 5 inches) of the device.
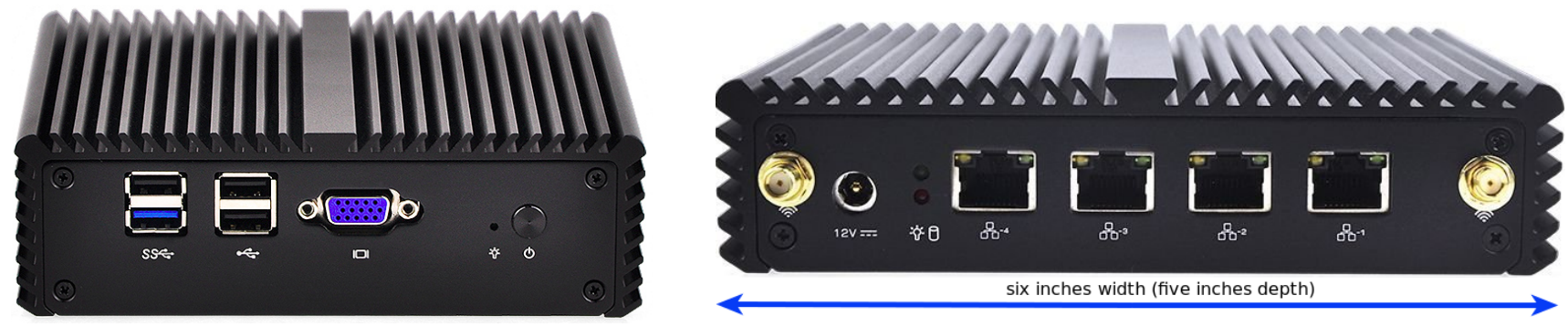

Figure 1. Front and back view of the Qotom Mini-PC.

It contains a Quad-Core Intel Celeron J100 CPU running at a nominal $2 \mathrm{GHz}$. This machine was selected because it had a small form factor chassis that blends seamlessly into network cabinets and similar environments. It has four LAN network interfaces (RJ-45 jacks). It is designed for 
advanced performance and built for multitasking. Table 1 shows the basic specification of the product.

Table 1. Qotom Mini-PC hardware specifications.

\begin{tabular}{|l|l|}
\hline CPU & $\begin{array}{l}\text { Intel Celeron Processor J1900 (Quad-Core 2M Cache, 2 GHz, up } \\
\text { to 2.42 GHz) }\end{array}$ \\
\hline Memory & 4GB RAM, 32GB SSD \\
\hline Architecture & 64-bit computing/addressing \\
\hline Network Cards & Four Interfaces, each one (1) Gbps \\
\hline
\end{tabular}

The Qotom's preloaded Operating System is PfSense [2]. This OS has excellent Firewall capabilities. However, Ubuntu's Linux-based v16.04 LTS 64-bit Server has robust Firewall capabilities and has excellent user forums and support. Therefore, Ubuntu [3] became the project OS.

Once the Qotom device was configured as a router and confirmed working, tests began. Three test categories total; two different measures of throughput, and one measure of data latency. Section VI shows side-by-side throughput-test comparisons for the Qotom-based home-built router and a set of commercial routers. Table 2 lists the names and models of the commercial routers and shows their publicly-reputed throughput rates.

Table 2. Commercial routers used for project comparison testing.

\begin{tabular}{|c|c|c|c|}
\hline $\begin{array}{c}\text { NETGEAR } \\
\text { R7000 } \\
1 \mathrm{Gbps}\end{array}$ & Linksys EA6900 & TP-Link Archer C9 & Linksys E900 \\
\hline
\end{tabular}

\section{OS Installation}

For most Linux users, the days of a CD or DVD based OS installation is gone. Two factors are key to this development. First, the presence of needed high capacity ( $1-2 \mathrm{~GB})$ pluggable USB memories. Second, the presence of PC system BIOS enabled boot from USB/Flash. Therefore, the OS install on the mini-PC is doable provided a bootable Ubuntu Install USB memory stick is available.

The install disk image (iso file) for Ubuntu 16.04 LTS 64-bit architecture server is on the Ubuntu website [4]. The workflow for installing an iso file onto a bootable USB memory stick is familiar to many.

Download iso install file onto an existing Ubuntu (or other) computer. Invoke a "startup" disk creator application. The disk creator uses the "*.iso" file as its input source. Disk creator uses the 2GB USB memory stick as its output target. The program completes. The result, the USB memory stick is bootable. 
Before installing the OS, it is necessary to attach peripherals to the mini-PC. Remove keyboard and monitor after successful OS install and router configuration. The peripherals and connections are listed below.

\section{VGA monitor (VGA Keyboard (USB USB memory stick Internet (network cable)} cable) cable)

Powering on the mini-PC, with USB memory stick in place, forces the system to boot from the stick. Installation proceeds using the monitor and the keyboard. The installation "target" is the Qotom 32GB internal solid state memory device. Installation steps include specifying a common desktop environment (e.g., Ubuntu Unity). The Ubuntu website has excellent installation support materials [5].

After setup, removal of the USB memory stick and reboot, Ubuntu is ready for use. If desired, configure Ubuntu for remote Secure Shell (ssh) or remote desktop protocol (RDP) connections. For usability, a remote connection has its advantages in a small lab. Doing so avoids working adjacent to the clutter of laboratory equipment.

\section{Basic Router Configuration}

The first step is to (1) retrieve necessary information concerning the integrated network interfaces cards (NIC) and (2) initialize the system to make those interfaces "router usable" at boot. This work requires working from the Linux Command Line Interface (CLI). The "ifconfig" command is used to query the system about the internal NIC cards. Below, an excerpt of the ifconfig report concerning the "loopback" interface:

lo Link encap:Local Loopback
inet addr:127.0.0.1 Mask:255.0.0.0
inet6 addr: ::1/128 Scope:Host
UP LOOPBACK RUNNING MTU:65536 Metric:1
RX packets:4567 errors:0 dropped:0 overruns:0 frame:0
TX packets:4567 errors:0 dropped:0 overruns:0 carrier:0
collisions:0 txqueuelen:1
RX bytes:599438 (599.4 KB) TX bytes:599438 (599.4 KB)

The device name is "lo." The IP address is 127.0.0.1, and the netmask is 255.0.0.0. The loopback device is a "virtual" device. No hardware is present. In the same manner, ipconfig reports all physical network interfaces information. The previously connected network interface device will have an active IP address and netmask values. The remaining unconnected interfaces will not report an IP address and netmask value.

The first two physical interface cards reported by ifconfig are (1) enp $1 \mathrm{~s} 0$ and (2) enp $2 \mathrm{~s} 0$. The enp1s0 device is the connection to the Internet (WAN). The enp2s0 will be the LAN side network interface. These are not initially present the "/etc/network/interfaces" file. They must be for future network operations. 
The initial /etc/network/interfaces file content is:

$$
\begin{aligned}
& \text { \# interfaces(5) file used by ifup( } 8) \text { and ifdown( }(8) \\
& \text { auto lo } \\
& \text { iface lo inet loopback }
\end{aligned}
$$

Notice the relative emptiness in the block above, only the loopback local interface (iface lo) device appears. It is missing the enp1s0 and enps 1 devices. Edit the enp $1 \mathrm{~s} 0$ and enps 1 devices into the file using a Linux editor. Shown in Figure 2 is the text editor "sublime" (subl). Any Linux text editor can be substituted. Figure 2 includes the edits in the /etc/network/interfaces file.

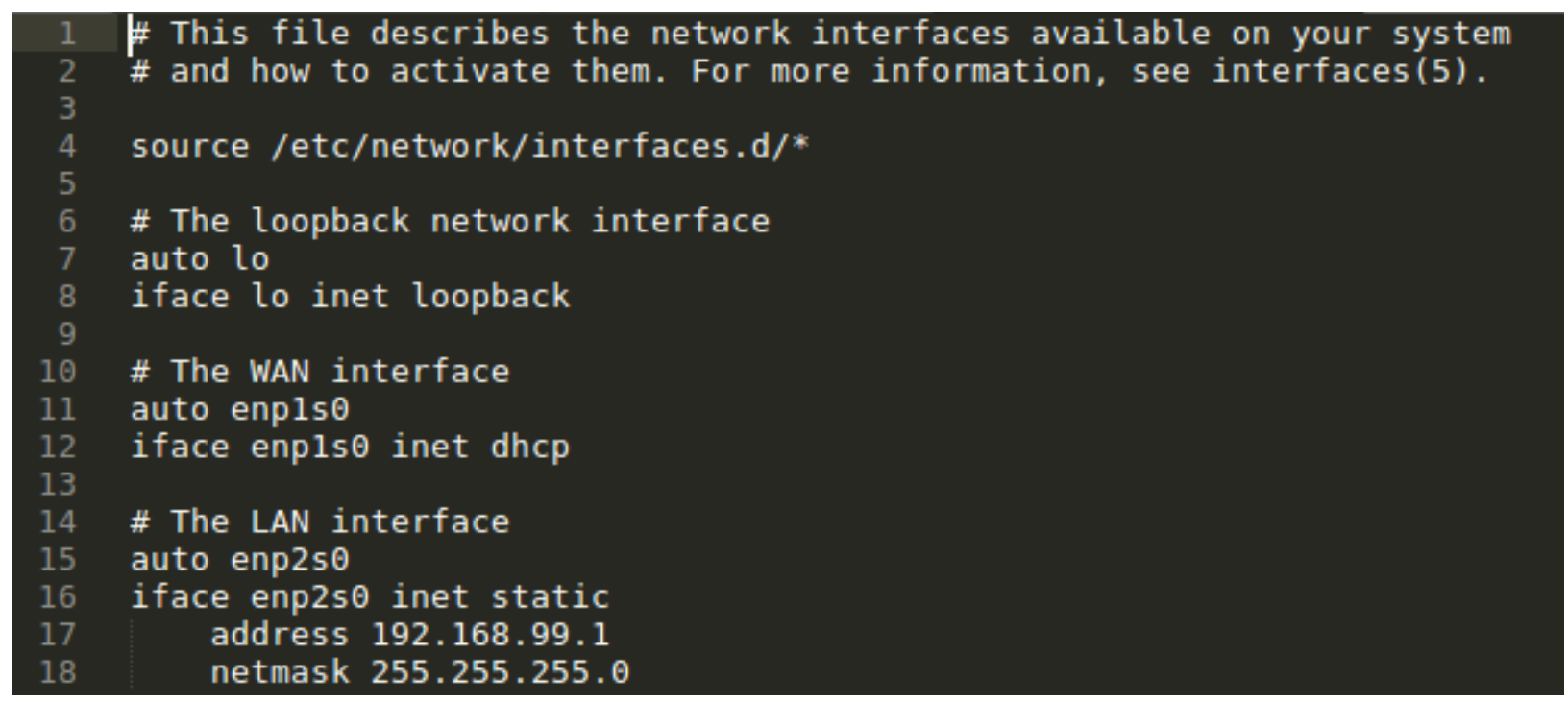

Figure 2. Edited WAN/LAN network interfaces in the Network Interface File.

The enp1s0 is the interface to the "outside" of the network, the WAN. The (editor created) additional /etc/network/interfaces text lines are (1) Auto enp1s0 and (2) iface enp1s0 inet dhcp. Adding these lines complete the enp1s0 device configuration.

The enp2s0 is the interface on the "inside" of the network, the LAN. The four (editor created) additional /etc/network/interfaces text lines are (1) Auto enp2s0, (2) iface enp2s0 inet static, (3) address 192.168.99.1, and (4) netmask 255.255.255.0. Adding these lines complete the enp1s0 device configuration. Now the interfaces are ready on every mini-PC router startup. Per the system directives, an external Dynamic Host Control Protocol (DHCP) server is expected to assign an IP address to the WAN interface (enp1s0). Static addressing assigns the fixed IP address to the LAN interface (enp2s0).

The next step was to enable forwarding of IP traffic between interfaces. Forwarding is a crucial step. Without this, the router will not work as required. The Linux network operations configuration file, "/etc/sysctl.conf" determines "forwarding" status (on/off). 
Uncommenting the one line from the file turns forwarding "on" for IPv4 traffic. All these changes are applied by saving the file and rebooting the system. Figure 3 shows the now uncommented "net.ipv4.ip_forward=1" line.

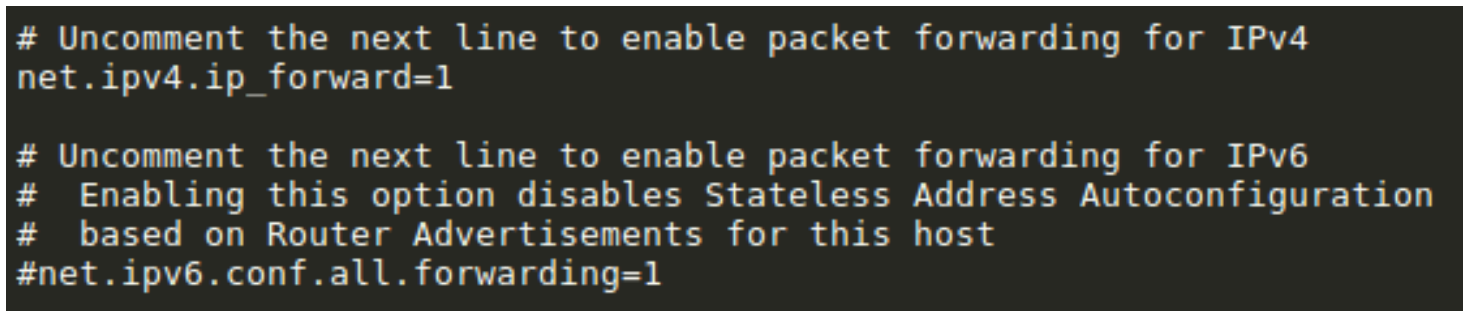

Figure 3. Forwarding IPv4 traffic enabled.

Testing proceeded after a required mini-PC router reboot. Network connectivity from WAN to LAN was confirmed.

\section{Network Configuration}

Next step, the network design work. Table 3 list the network design specifics for the router.

Table 3. Network design of router.

\begin{tabular}{|l|l|}
\hline \multicolumn{1}{|c|}{ Subject/Task } & \multicolumn{1}{c|}{ Solution/Parameter } \\
\hline WAN IP address of router & Dynamic Host Control Protocol (DHCP) \\
\hline LAN IP address of router & 192.168 .99 .1 \\
\hline LAN client IP addresses & Dynamic Host Control Protocol (DHCP) \\
\hline Number of hosts allowed on LAN and LAN Mask & 100 hosts $\quad$ Mask of LAN 255.255.255.0 \\
\hline IP address range of hosts on LAN & 192.168 .99 .100 to 192.168.99.199 \\
\hline Remapping Method WAN to LAN & Network Address Translation (NAT) \\
\hline IP to Fully Qualified Domain Name translation & DNS Server \\
\hline
\end{tabular}

There are many DHCP server packages available online however the most common and stable is the ISC DHCPD. This server quickly installs from the terminal with the following command.

$$
\text { sudo apt-get install isc-dhcp-server }
$$

Once installed, the DHCP configuration file (dhcpd.conf) must be modified. It must contain subnet-specific information (Table 3). The Linux editor "vim" is used. Any Linux text editor can be substituted. 


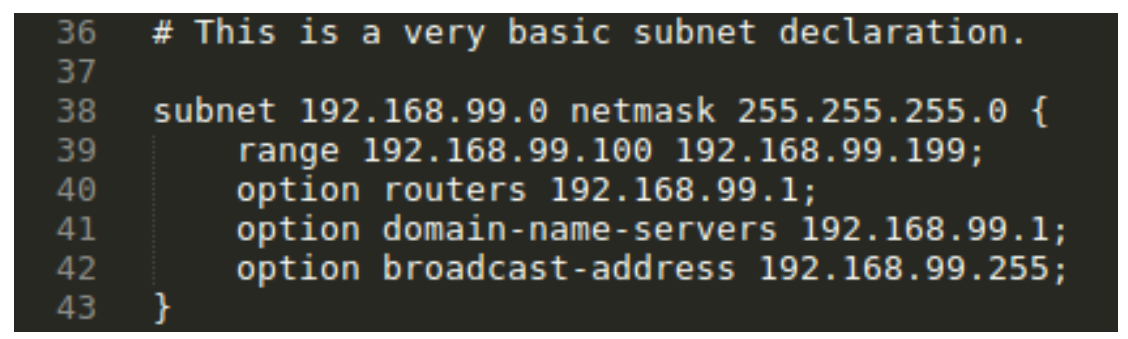

Figure 4. IP address declarations in the dhcpd.conf file.

Figure 4 shows the edited dhcpd.conf file. The design values shown are from Table 3 . The chosen router network IP address of 192.168.99.1 is arbitrary. The chosen span of 100 hosts is arbitrary. Addresses run between 192.168.99.100 and 192.168.99.199. After making the changes, manually restart the isc-dhcpd server:

sudo /etc/init.d/isc-dhcp-server restart

The Domain Name System (DNS) server install is even easier; this will allow access to websites, such as www.google.com, without the need of, or knowledge of, the website's numeric dotted decimal IP address.

sudo apt-get install bind9 bind9utils bind9-doc

\section{Firewall Network Configuration}

The primary network configuration in the previous section included only one "routing" directive. That directive was "forwarding enabled." Stopping network feature development work at this point yields an extremely insecure and dangerous network. The next work will use a network firewall feature known as "iptables" [6] It establishes network traffic operating rules. The indepth knowledge of iptable rule creation is beyond the scope of this work. However, discussion includes background information.

Good network administration practice requires "iptables" rules restoration from a saved configuration. A script file restores rules at boot. The "flow" of network startup operations ensures enforcement of rules before network interfaces are enabled. Figure 5 shows the creation of the "restoration" iptables script file used at boot. Figure 6 shows the /etc/network/iptables file content used in this project. The content determines the iptable rule application structure.

sudo vim /etc/network/if-pre-up.d/iptables

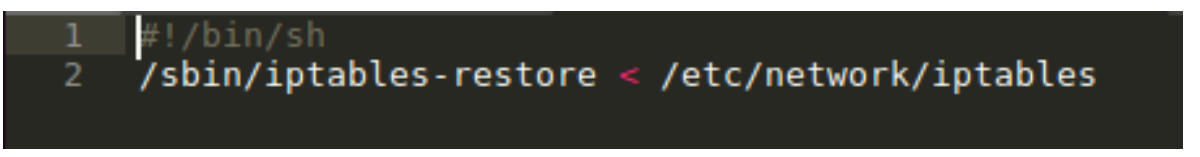

Figure 5. Creation of restoration script file from /etc/network/if-pre-up.d/iptables. 
System "housekeeping" requires execution of the next Linux Command Lines. They ensure needed file ownership and permissions for the script file /etc/network/if-pre-up.d/iptables.

sudo chown root /etc/network/if-pre-up.d/iptables

sudo chmod 755 /etc/network/if-pre-up.d/iptables

Assuming the /etc/network/iptables file exists, its basic rules are in the "saved configuration" iptables file at boot.

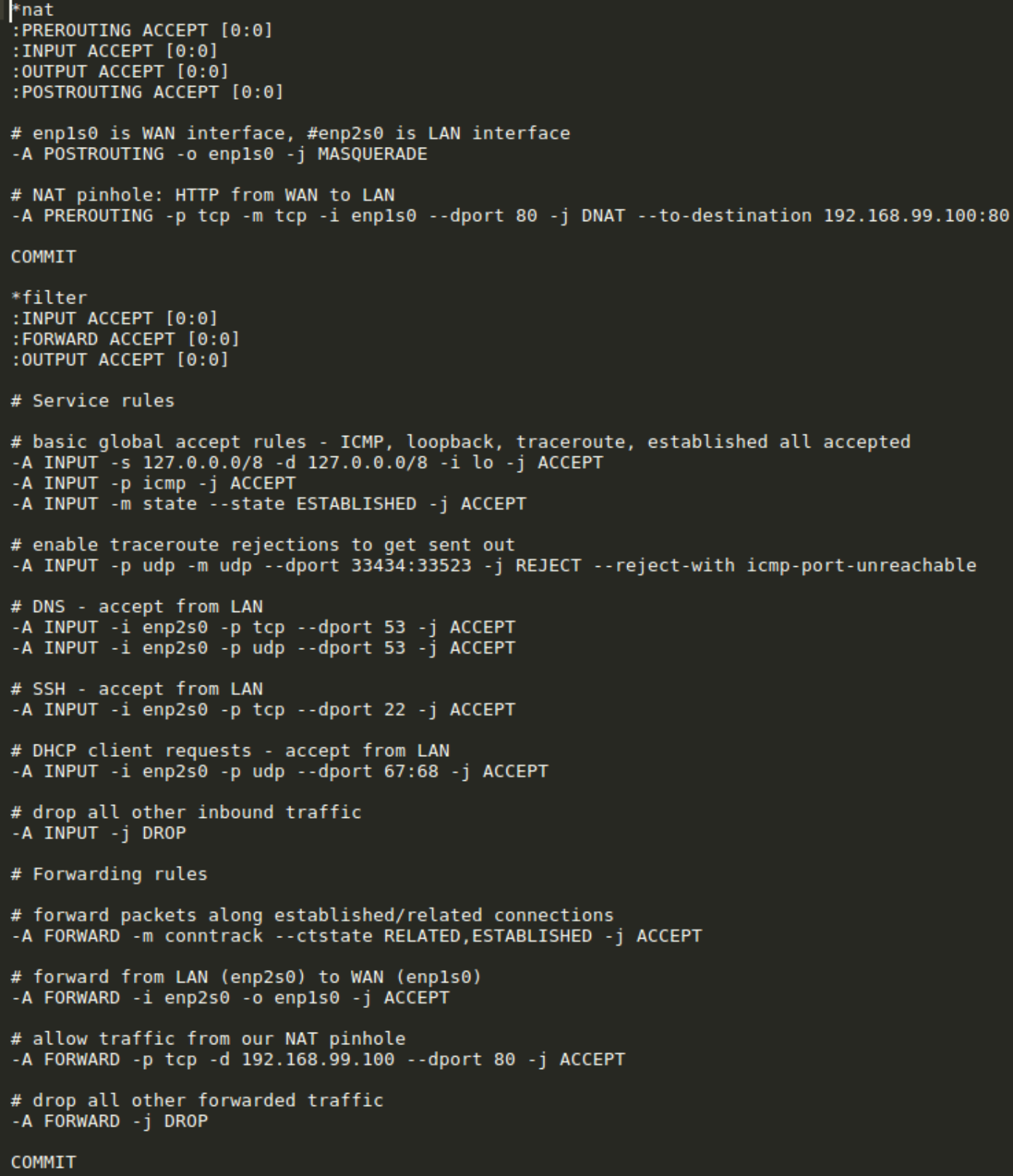

Figure 6. Rule contents of the /etc/network/iptables file. 
As stated earlier, the in-depth knowledge of iptable rule creation is beyond the scope of this work. However, the general form, shown in Figure 6, is (1) start of a table denoted by name and (2) end of a table denoted by COMMIT. Above the COMMIT statement, reside any IP traffic handling rules. Each table contents contain "chains." Rules belong to chains.

Two iptables tables exist in Figure 6, (1) the Network Address Translation table named "nat," and the global/general table named "filter." Shown below, left, is the "nat" table chains. Shown below, right, is the "filter" table chains.

$$
\begin{aligned}
& \text { :PREROUTING ACCEPT [0:0] } \\
& \text { :INPUT ACCEPT[0:0] } \\
& \text { :OUTPUT ACCEPT [0:0] } \\
& \text { :POSTROUTING ACCEPT [0:0] }
\end{aligned}
$$

$:$ INPUT ACCEPT $[0: 0]$
$:$ FORWARD ACCEPT [0:0]
$:$ OUTPUT ACCEPT $[0: 0]$

Highlighted rows indicate chains that are filled out by rule statements in Figure 6 . The other chains do not have rule statements. The rules begin with the "-A" symbol. All IP traffic navigates the iptables structure for rule testing, as indicated in Figure 7. The nat table rules are specific to acting on Network Address Translation directives. The filter table's principle role is security. That is, determining whether to drop network packets or not.

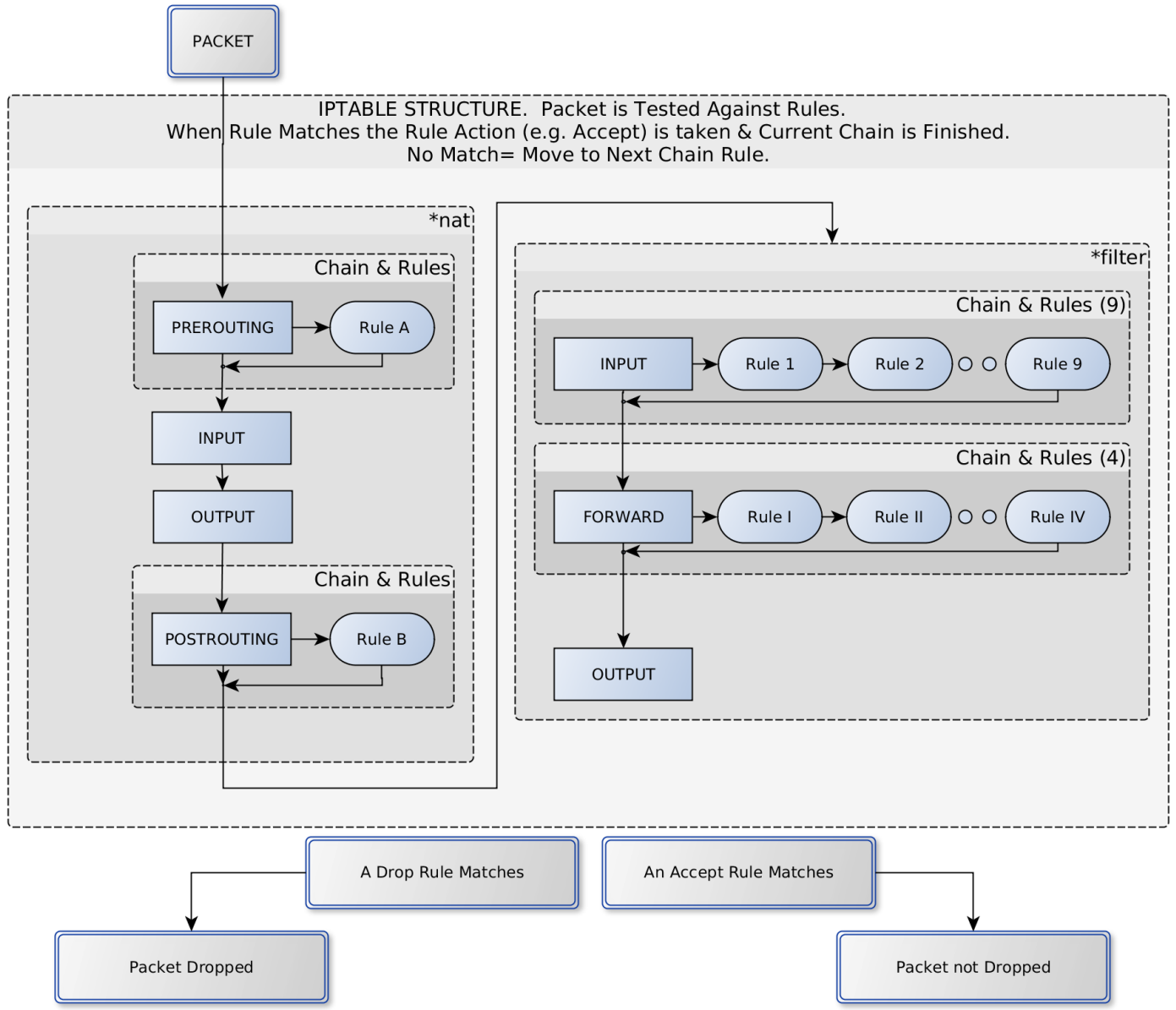

Figure 7. IP traffic navigation using the IPTABLE structure. 
Figure 6 shows the Filter rules (navigated as shown in Figure 7), they represent excellent networking practices. Here are two examples (Table 4 and Table 5):

Table 4. A Postrouting chain and rule in an IPTABLE structure.

The "Postrouting" chain (*nat) contains the following rule:

-A POSTROUTING -o enp1s0 -j MASQUERADE

Which is interpreted as: replace the LAN IP address of a host with the WAN IP address of the mini-PC router. Output network interface is the WAN port. It is an unconditional rule.

Table 5. An Input chain and rule in an IPTABLE structure.

The "Input" chain (*filter) contains the following rule:

The -A INPUT -i enp2s0 -p tcp --dport 22 -j ACCEPT

Which is interpreted as: the SSH port 22 accepts traffic from the mini-PC router's LAN network interface. SSH connections are accepted. Therefore, SSH service is available.

\section{Testing}

There are many possible ways to test a router, and there are benchmarks to consider. For home use, most customers do not have network connect speeds exceeding $100 \mathrm{Mbps}$ [7]. Most commercially available routers can easily meet these connection speeds. However, in business applications, higher capacities are required.

The first measurement considered by many is throughput. The chosen network tool "iperf" [8] tested channel throughput. Figure 8 shows the test setup, with the mini-PC router in place. Testing included the commercial routers.

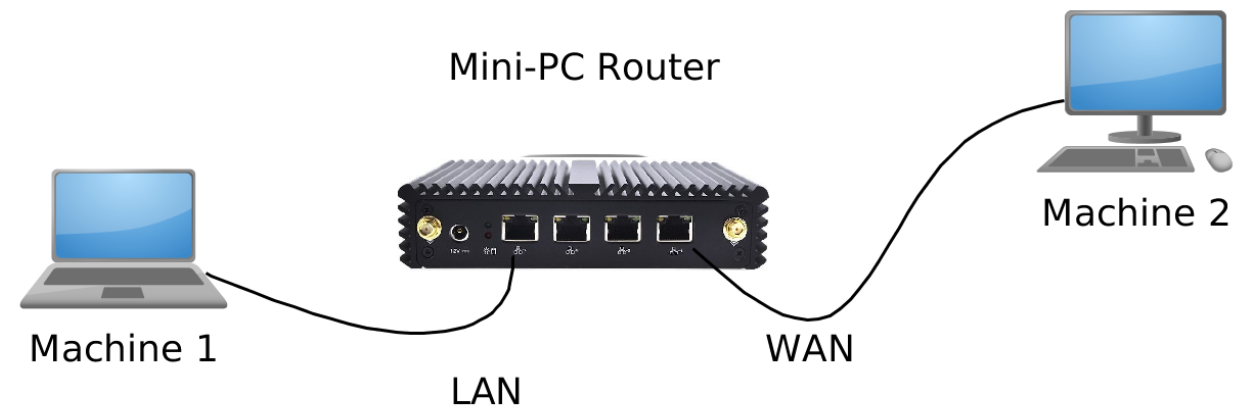

Figure 8. Network measurement testbed. 


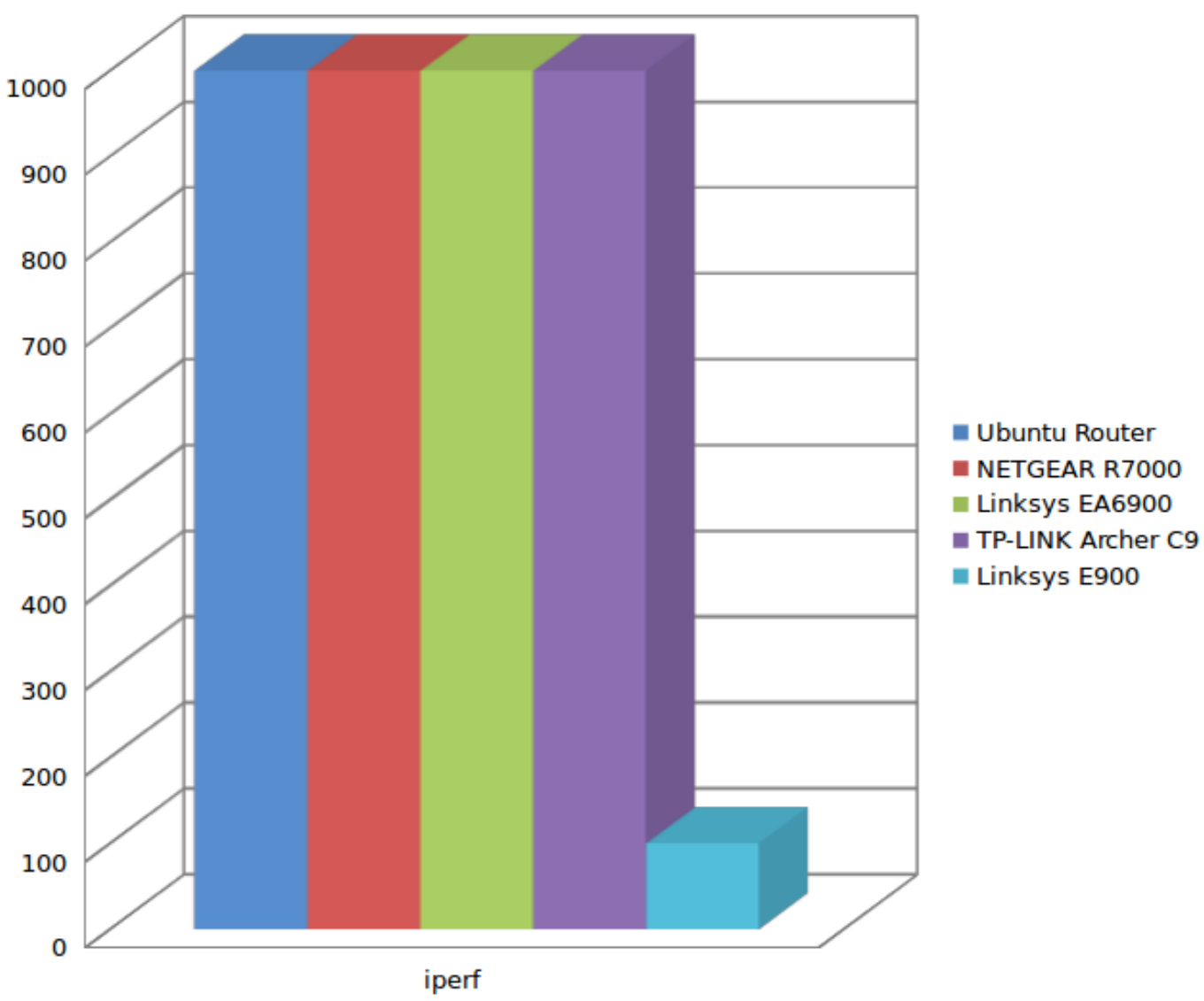

Figure 9. "Iperf" router throughput (Mbps) test results (chart).

The recorded test throughput of each commercial device equaled their advertised throughput. The Ubuntu-based mini-PC router's test result equaled the advertised throughput ratings of their network interfaces. See Figure 9. These results show that the Iperf throughput test does not provide the full picture. Another common statistic Internet users look at is latency. Tests showed latency for all devices were less than one (1) $\mathrm{mS}$. This latency is well below other latencies found in typical network paths. Therefore, the latency values seen are acceptable for all routers tested.

The final test set used the free network measurement tool LANBench [9]. This product tests unidirectional traffic as well as bidirectional traffic. Deploy LANBench on both machines. Machine 2 acted as the server (see Figure 8). Machine 1 acted as the client. Figure 10 shows the server user-interface window at Machine 2. 


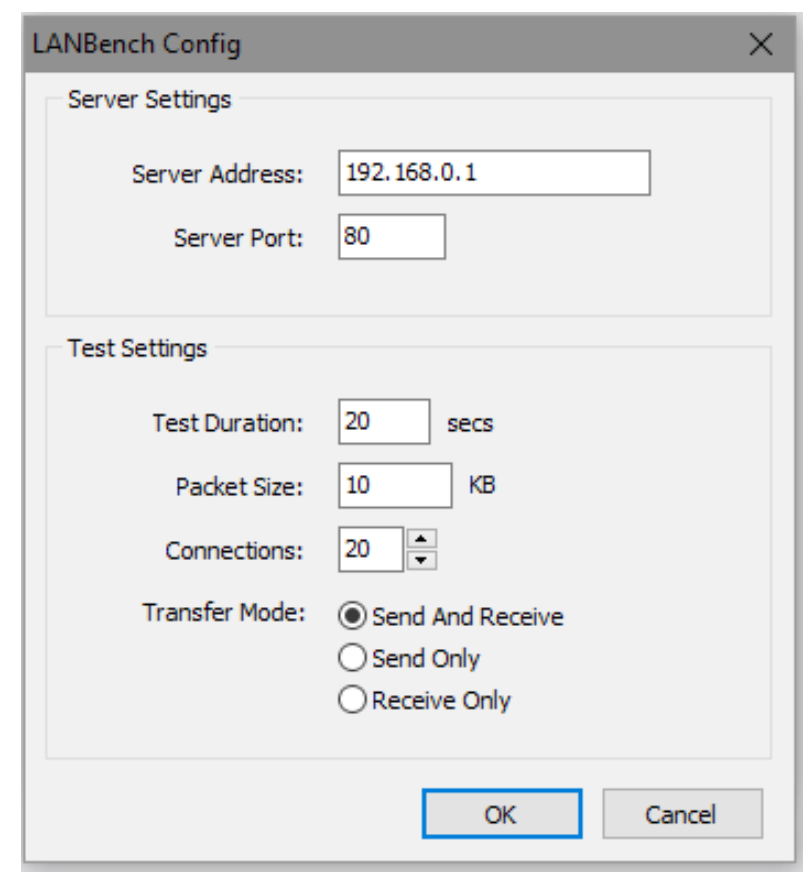

Figure 10. LANBench server graphic user interface.

All transfer modes were exercised for each router; repeated five times on each router tested. Packet size set at $10 \mathrm{~KB}$. Test duration set at 20 seconds. Table 6 shows the calculated averages. Figure 11 charts the same data.

Table 6. Calculated average data rates for LANBench test.

\begin{tabular}{|c|c|c|c|c|c|}
\hline Test Description & $\begin{array}{c}\text { Ubuntu } \\
\text { Router }\end{array}$ & $\begin{array}{c}\text { NETGEAR } \\
\text { R7000 }\end{array}$ & $\begin{array}{c}\text { Linksys } \\
\text { EA6900 }\end{array}$ & $\begin{array}{c}\text { TP-LINK } \\
\text { Archer C9 }\end{array}$ & Linksys E900 \\
\hline WAN -> LAN & $939.6 \mathrm{Mbps}$ & $931.4 \mathrm{Mbps}$ & $923.6 \mathrm{Mbps}$ & $756.0 \mathrm{Mbps}$ & $94.1 \mathrm{Mbps}$ \\
\hline LAN -> WAN & $957.2 \mathrm{Mbps}$ & $941.7 \mathrm{Mbps}$ & $844.2 \mathrm{Mbps}$ & $755.9 \mathrm{Mbps}$ & $94.0 \mathrm{Mbps}$ \\
\hline $\begin{array}{c}\text { Total } \\
\text { Simultaneous }\end{array}$ & $1463.3 \mathrm{Mbps}$ & $1378.3 \mathrm{Mbps}$ & $1135.1 \mathrm{Mbps}$ & $1189.7 \mathrm{Mbps}$ & $149.6 \mathrm{Mbps}$ \\
\hline
\end{tabular}




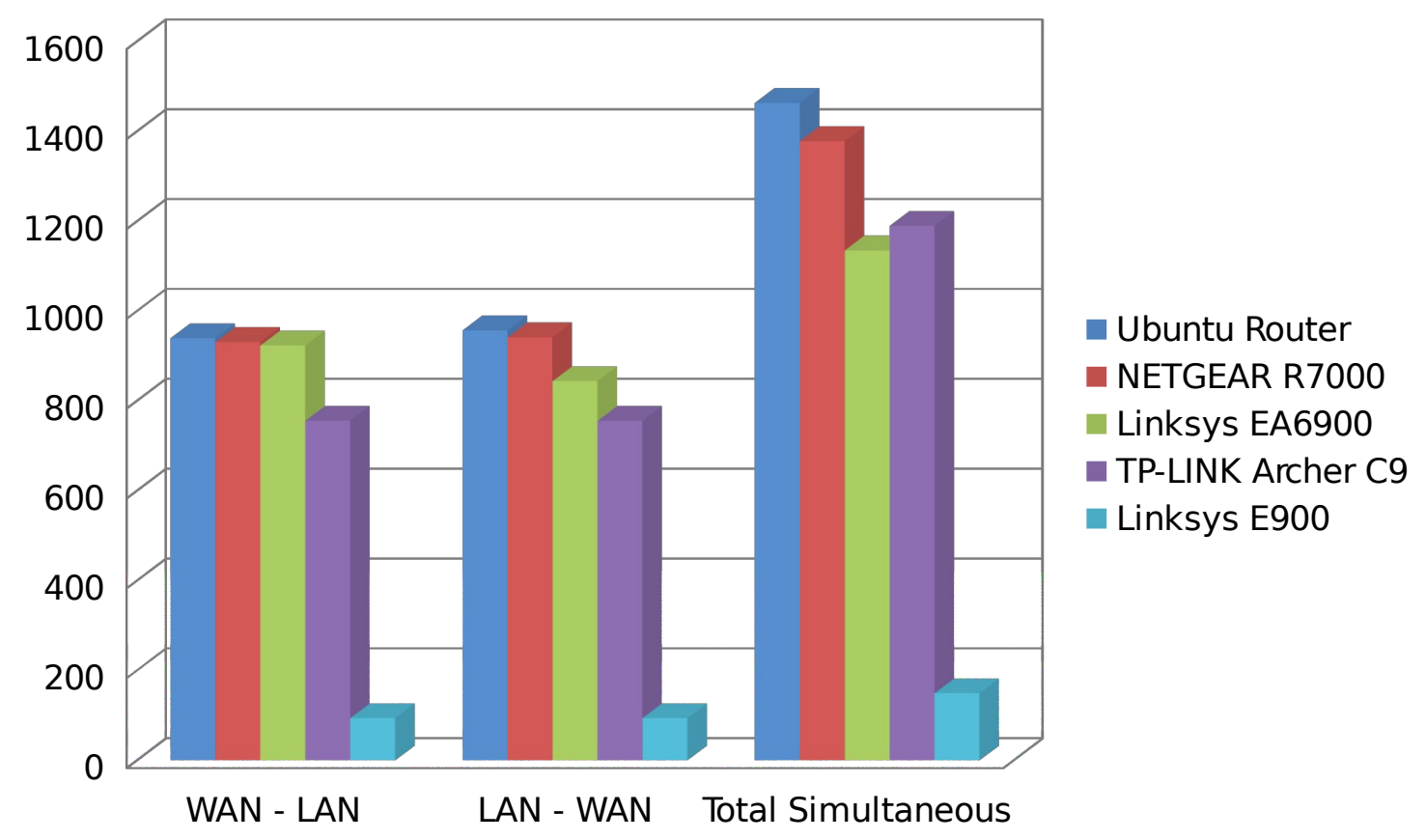

Figure 11. Calculated average data rates (Mbps) for LANBench test (chart).

\section{Conclusion}

The conversion of the Qotom mini-PC from a PfSense OS to Ubuntu OS went without difficulty. Organizational skills are required; stepping through the creation of an Ubuntu boot installation USB memory stick ( 2 GB stick) requires attention to details. The boot install requires plugging in a USB keyboard and the borrowing of a VGA monitor. Once Ubuntu is installed, work proceeds centered on installing and configuring networking features. First router achievement was the creation of an "unprotected" router. Creating a Firewall is more challenging because of the nuances of the Iptables network feature. However, use of standard Iptable tables, chains, and rules statements makes the task easier.

The custom built router consistently performed well against a set of commercially available routers. The results make it apparent that the mini PC router can efficiently compete with those routers. The unidirectional results make it apparent that the device is operating near the limits of its hardware network interfaces. Further, Ubuntu's ability to process network transactions are not a limiting factor in the tests. Increasing mini-PC memory is an option if real-life results indicate the router is operating below test results.

The price of the Qotom mini-PC was \$200. The price of the commercial routers, when new, was near $\$ 200$. The laboratory/classroom educational potential is present in the mini-PC. The Linux world holds a remarkable market share of the server industry. Learning the Linux Command Line Interface, the network connections, and Linux-based network administration tools have advantages. Its educational potential makes it well suited for use in senior projects and special projects. 


\section{References}

1. “Qotom Technology Company, LTD.”, Q190G4N Mini PC 4 LAN sales information, [online] Available: http://www.qotom.net/goods-133-Q190G4N+Mini+PC+4+LAN.html

2. "Netgate", pfSense open source firewall project, [online] Available: https://www.netgate.com/solutions/pfsense/features.html

3. "Ubuntu", Ubuntu main web page, [online] Available: https://www.ubuntu.com/

4. "Ubuntu”, Ubuntu 16.04 Server Installation Download, [online] Available:

https://www.ubuntu.com/download/serverReferences

5. "Ubuntu”, Ubuntu Community Support, [online] Available:

https://www.ubuntu.com/support/community-support

6. "Ubuntu", Ubuntu Firewall Documentation Iptables, [online] Available:

https://help.ubuntu.com/community/IptablesHowTo

7. "Statista Inc.", Average internet connection speed in the United States from 2007 to 2017 (in Mbps), by quarter, [online] Available: https://www.statista.com/statistics/616210/averageinternet-connection-speed-in-the-us/

8. "Wikipedia", Iperf Wikipedia Entry, [online] Available: https://en.wikipedia.org/wiki/Iperf

9. Saw, Z., LANBench, [online] Available: http://www.zachsaw.com/?

pg=lanbench tcp network benchmark 